\title{
Sleep Fragmentation Induces Activation of NOD-Like Receptor Protein-3 Inflammasome in Rat Hippocampus
}

\author{
Dae Wui Yoon, $\mathrm{PhD}^{1}$, Sungjin Shin², Jinny Choi², Claire Shin², Chris Lee², Jenna Lee², Huck-Jun Hong ${ }^{2}$, Richard Jo², \\ Tommy Lee ${ }^{2}$, Jeff Kim², Nuri Jun, MS ${ }^{3}$, Seung Ku Lee, PhD¹, Jinkwan Kim, PhD ${ }^{4}$, Hoon-Jung Jwa, MD, PhD \\ ${ }^{1}$ Institute of Human Genomic Study, College of Medicine, Korea University Ansan Hospital, Ansan, Korea \\ ${ }^{2}$ Asia Pacific International School, Seoul, Korea \\ ${ }^{3}$ Department of Foods and Nutrition, College of Natural Sciences, Kookmin University, Seoul, Korea \\ ${ }^{4}$ Department of Biomedical Laboratory Science, College of Health Science, Jungwon University, Goesan, Korea \\ ${ }^{5}$ Seoul Jung-Tong Clinic, Seoul, Korea
}

Background and Objective Continuous sleep is important for cognitive function; studies have shown that disrupted sleep has a deleterious effect on hippocampus-dependent cognitive function, but the exact mechanism is unknown. The inflammasome NOD-like receptor protein-3 (NLRP-3) has been reported to cause memory and cognitive dysfunction, but no studies have examined whether NLRP-3 is up-regulated in the hippocampus by sleep fragmentation (SF). Therefore, we investigated whether SF affected NLRP-3 activation in the rat hippocampus in a wheel-based SF model.

Methods We randomly divided 7-week-old male Wistar rats into 4-day SF, 4-day exercise control (EC), 8-day SF, and 8-day EC groups. SF was accomplished with a forced walking wheel with a 30 -s on/90-s off cycle, and we set EC at $10 \mathrm{~min}$ on/30 min off. We performed Western blots to compare NLRP-3 expression levels, and we measured malondialdehyde (MDA) levels in the hippocampus with a commercial kit.

Results The SF group had more but shorter NREM bouts. Western blot analysis revealed that 4 and 8 days of SF up-regulated the expression of the NLRP-3 complex in the hippocampus including ASC, caspase-1, and IL- $1 \beta$. Thioredoxin-interacting protein expression also increased more in the SF group, and the MDA level increases were greater in the SF groups than in the corresponding EC groups.

Conclusions SF up-regulates NLRP-3 in the hippocampus. Additional behavioral and mechanistic studies are required to clarify the role of NLRP-3 in the hippocampi of rats subjected to SF.

Sleep Med Res 2017;8(1):26-32

Key Words Sleep fragmentation, NLRP-3, Hippocampus, Malondialdehyde.

Received: May 8, 2017

Revised: June 8, 2017

Accepted: June 14, 2017

\section{Correspondence}

Hoon-Jung Jwa, MD, PhD

Seoul Jung-Tong Clinic, 1 Nangye-ro 23-gil,

Jung-gu, Seoul 04573, Korea

Tel +82-2-2252-0075

Fax +82-2-2254-0075

E-mailiisolevo@naver.com

\section{INTRODUCTION}

Sleep is essential for maintaining normal body function. The appropriate amount of sleep is important, but the continuity of sleep is also important. ${ }^{1}$ Sleep fragmentation (SF) can be defined as disrupted sleep with abrupt changes in electroencephalogram (EEG) during sleep, which is known as sleep arousal. SF can be caused by internal factors (e.g., sleep apnea, restless leg syndrome, and pain) or external factors such as noise and temperature. ${ }^{2,3}$ Clinically, SF has been reported to occur at high frequency in patients with osteoarthritis and arthritis, and frequent $\mathrm{SF}$ is an important factor in reducing sleep quality in these patients. In particular, SF is common in patients with obstructive sleep apnea (OSA) $)^{4}$ and frequently occurs in patients with lung diseases such as chronic obstructive pulmonary disease, nighttime asthma, narcolepsy, and Parkinson's disease. SF causes a variety of clinical problems; in animal and human studies, acute and chronic SF are associated with increased blood pressure, vascular endothelial dysfunction and changes in blood vessel structure, increased resistance to leptin and insulin, weight gain, decreased blood-brain barrier function, and cancer cell growth.,

Inflammasomes are multi-protein complexes that play a key role in the innate immune sys- 
tem. They comprise an intracellular sensor, a NOD-like receptor (NLR), procaspase-1, and ASC, an adapter protein. ${ }^{7}$ Inflammasome activation promotes autocatalytic activation of a cysteine protease, caspase- 1 , which activates the cytokines pro-IL-1 $\beta$ and IL-18. NLR protein-3 (NLRP-3) is a representative inflammasome that consists of NLRP-3, ASC, and pro-caspase-1; this complex is essential for producing mature IL- $1 \beta$ in response to a variety of signals. NLRP-3 is activated by danger-associated molecular patterns, including endogenous risk signals such as bacterial toxins, pathogen-associated molecular patterns, and adenosine triphosphate (ATP). ATP outside the cell binds to the $\mathrm{P} 2 \mathrm{X} 7$ receptor and promotes $\mathrm{K}^{+}$release. This $\mathrm{K}^{+}$release reduces intracellular $\mathrm{K}^{+}$levels, which induces oligomerization of NLRP-3 and activates it. ${ }^{8}$ NLRP- 3 can also be activated by other endogenous stress-related risk signals such as monosodium urate crystals, $\beta$-amyloid, and reactive oxygen species (ROS) ${ }^{9-11}$ NLRP3 -dependent IL-1 $\beta$ has been reported to contribute to the development of insulin resistance ${ }^{12}$ and is associated with impaired cognitive function. ${ }^{13,14}$

SF resulted in cognitive and memory dysfunction by increasing nicotinamide adenine dinucleotide phosphate oxidase activity $^{15}$ and down-regulating the proteins that account for synaptic neurotransmission. ${ }^{16}$ However, the exact mechanism by which SF impairs cognitive and memory function remains unresolved. Because IL- $1 \beta$ is associated with cognitive impairment and because IL-1 $\beta$ expression and its activator can be up-regulated in stress conditions, we hypothesized that SF could increase IL-1 $\beta$ levels in the hippocampus by activating NLRP-3 inflammasome. That is, increased IL-1 $\beta$ might have an important role in memory and cognitive impairment. To test this hypothesis, we investigated whether SF activated NLRP-3 inflammasome in the hippocampus and the possible mechanisms of this activation.

\section{METHODS}

\section{Animals}

We used 7-week-old male Wistar rats (Orient Bio, Seongnam, Korea) in this study. All animals were maintained in a temperature-controlled facility with alternating 12 -h cycles of light and dark (light on at 8:00 am); the animals had free access to food and water. We randomly assigned 48 rats to one of the following groups: 4 days of SF ( $n=12), 8$ days of SF $(n=12), 4$ days of exercise control (EC; $n=12)$, and 8 days of EC group $(n=12)$. We also used an additional 10 rats for EEG measurements during $\mathrm{SF}(\mathrm{n}=5)$ and EC $(\mathrm{n}=5)$.

\section{Sleep Recording and Analysis Conditions}

We recorded sleep EEGs to evaluate the adequacy of the sleep segmentation model. We implanted two pairs of EEG and one pair of electromyography (EMG) electrodes in the rats, using isoflurane (Baxter, San Juan, Puerto Rico, USA) as an inhala- tion anesthetic. The induction concentration was $5 \%$, and the maintenance concentrations were $2-2.5 \%$. We inserted a pair of EEG electrodes (Pinnacle Technology Inc., Lawrence, KS, USA) into the frontoparietal and lateral lobes and a pair of EMG electrodes into the neck muscles, fixing them with a non-absorbable seal. After we connected the head mount to the electrodes, we firmly fixed them to the rat's head with dental acrylic. We sutured the incisions and administered antibiotics intraperitoneally for three days after surgery to prevent inflammation. After the operation, we dressed the wound area for several days and obtained a sleep EEG after 14 days. We amplified EEG and EMG signals with a preamplifier (Pinnacle Technology) and Sirenia software (Pinnacle Technology) to analyze the sleep stage. We divided sleep into three stages, awake, non-rapid eye movement (NREM), and rapid eye movement (REM), according to the amplitude and spectral analysis of the EEG and EMG waveforms; we set the duration of one epoch at 10 seconds. We recorded and analyzed 24 hours of EEG at baseline and after 4 or 8 days of SF or EC. We defined the frequency range for each waveform as delta $0.5-4 \mathrm{~Hz}$, theta $4-8 \mathrm{~Hz}$, and alpha $8-13 \mathrm{~Hz}$. We analyzed the percentage of sleep time by stage, number of bouts, and length of bouts during the entire recording time, defining about as at least two consecutive 10-s epochs for a given state that ended with any single state-change epoch.

\section{SF and EC Conditions}

We used a rat forced-locomotion wheel system (80805A; Lafayette Instrument Company, Lafayette, IN, USA) for SF and EC. For SF, the wheel-based device was operated with a $90 \mathrm{sec}$ off/30 sec on cycle for $6 \mathrm{~h} /$ day to mimic the arousal frequency of severe OSA. The speed was $4 \mathrm{~m} / \mathrm{min}$ considering that the average speed of a rat is $7 \mathrm{~cm} / \mathrm{s}$. The conditions for EC were 10 $\min$ on/30 min off for $6 \mathrm{~h} /$ day, which allowed for longer periods of undisturbed sleep. SF and EC were performed from 10:00 am to 4:00 pm during the entire experimental periods.

\section{Western Blotting}

After 4 or 8 days of SF or EC, the mice were anesthetized with $5 \%$ isoflurane, and their hippocampi were collected on ice. The tissue was immediately immersed in liquid nitrogen and stored at $-80^{\circ} \mathrm{C}$. The hippocampal tissues were homogenized in RIPA buffer (Sigma, St. Louis, MO, USA) supplemented with protease inhibitors. We quantified total protein concentrations with a BCA assay kit (Pierce, Rockford, IL, USA). For Western blotting, we loaded $50 \mu \mathrm{g}$ onto a $10 \%$ SDS-PAGE gel and then transferred the protein to a PVDF membrane at $300 \mathrm{~mA}$ for 1 hour. After electrophoresis, we confirmed the protein bands by Ponceau $S$ staining. We washed the membranes with Tris-buffered saline with Tween 20 and blocked them with 5\% skim milk for 1 hour to block nonspecific reactions. We diluted primary antibodies as follows: rabbit polyclonal anti-caspase-1 (1:1000; Abcam, Cambridge, MA, USA), cleaved caspase-1 (1:500; Novus Biologicals, 
Littleton, CO, USA), rabbit polyclonal anti-ASC (1:1000; Abcam), rabbit polyclonal anti-NLRP-3 (Abcam), rabbit polyclonal anti-thioredoxin-interacting protein (TXNIP) (1:1000; Santa Cruz Technologies, Dallas, TX, USA), and rabbit polyclonal anti-IL-1 $\beta$ (1:500; Abcam). The secondary antibody was goat anti-rabbit HRP-conjugated antibody in 5\% skim milk at 1:5000 for 1 hour at room temperature. We used $\beta$-actin (1:20,000; Bioworld Technology, St. Louis Park, MN, USA) as an internal control. We detected the signals with an ECL solution. We then developed the protein bands on X-ray film and converted them into image files with a scanner, and we quantified the band density with ImageJ. We normalized the intensity of each protein band to $\beta$-actin.

\section{Lipid Peroxidation}

We quantified the malondialdehyde (MDA), a product of lipid peroxidation in hippocampal tissue, with a commercial kit (OxiSelect TBARS assay kit; Cell Biolabs, San Diego, CA, USA) according to the manufacturer's instructions.

\section{Statistical Analysis}

We performed either paired $t$ tests or independent $t$ tests of the sleep EEGs as appropriate. We conducted all statistical analyses with SPSS version 20.0 (IBM, Chicago, IL, USA) and considered $\mathrm{p}<0.05$ significant.

\section{RESULTS}

\section{Wheel-Based System Effectively Fragmented Sleep in Rats}

Both the SF and EC groups showed significantly higher percentages of time awake at day 4 than at baseline (Fig. 1A), although the amount of time spent awake did not differ significantly between the groups. Throughout the experiment, the percentages of time spent in NREM and REM sleep did not differ significantly between the SF and EC groups or between baseline and follow-up (Fig. 1B and C). The SF group had more NREM but shorter bouts, which indicated highly fragmented NREM sleep (Fig. 2). The EC group had no significant difference in number of NREM bouts or NREM bout length compared with baseline, implying preserved sleep continuity in the EC group.

\section{SF Induced Up-Regulation of the NLRP-3 Complex}

We examined the expression levels of NLRP-3 and associated proteins by Western blot (Fig. 3A and C), and NLRP-3 expression was highest in the 4-day SF group. In the 8-day SF group, NLRP-3 expression was slightly lower than in the 4-day SF group but higher than in the 4-day and 8-day EC groups. Expression patterns of caspase- 1 and ASC, which form the NLRP-3 complex, were similar to that of NLRP-3. Levels of mature IL-1 $\beta$,
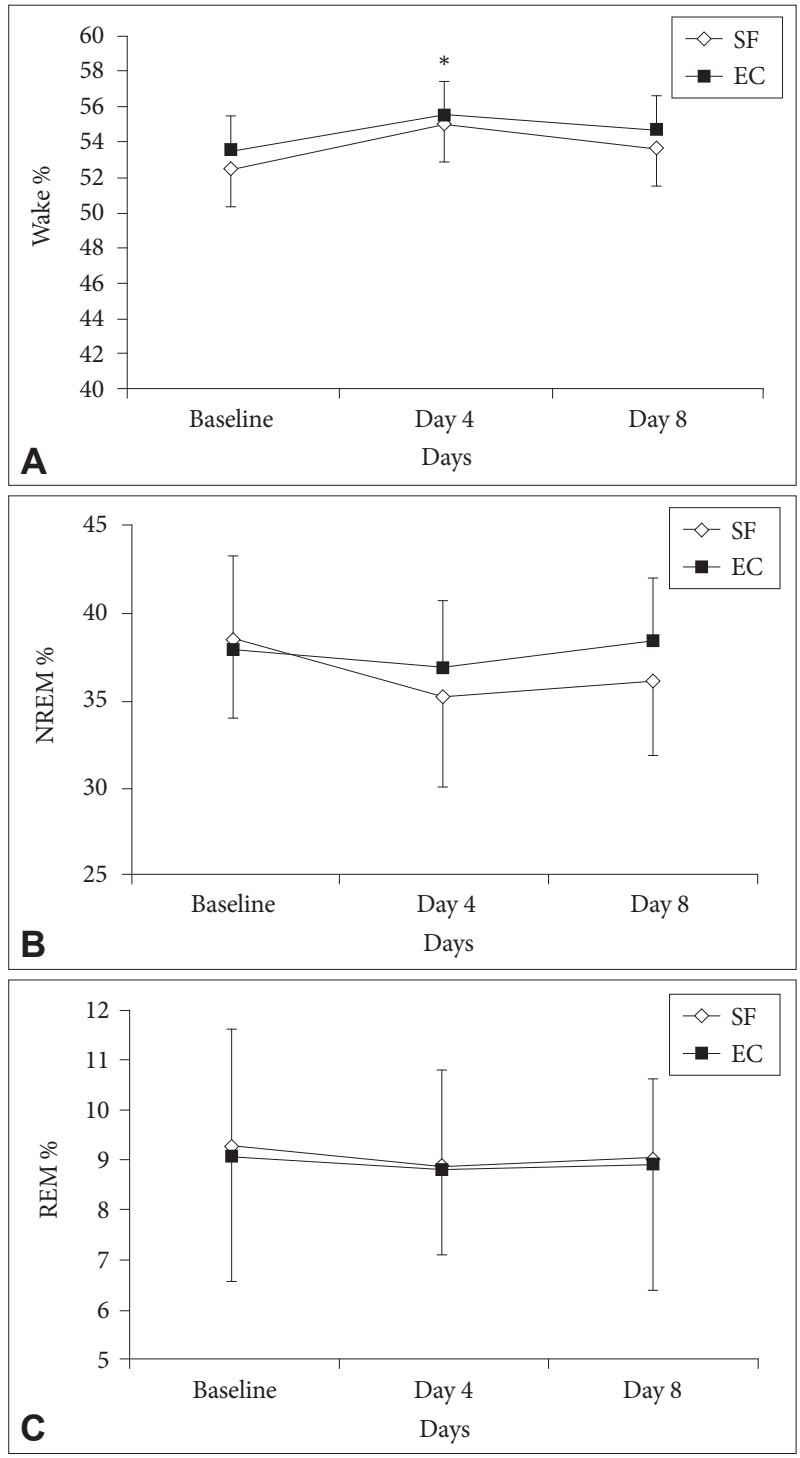

Fig. 1. Time spent awake (A) and in NREM (B) and REM (C) sleep in the SF and EC groups at baseline and on days 4 and 8 of SF or EC ( $n=5$, each group). Data are expressed as mean \pm SEM. ${ }^{*} p<$ 0.05 compared with baseline. SF: sleep fragmentation, EC: exercise control, NREM: non-rapid eye movement, REM: rapid eye movement.

which is generated by NLRP-3 activation, were significantly higher in the 4-day and 8-day SF groups than in the corresponding EC groups, and expression of cleaved caspase-1, which indicated caspase-1 activation, showed the same pattern. Because it is known that sleep disturbance may increase ROS, we examined the expression of TXNIP, which binds NLRP-3 and activates it under oxidative stress conditions. ${ }^{17}$ TXNIP expression was also higher in the SF groups than the EC groups (Fig. 3B and C).

\section{SF Increased Lipid Peroxidation}

Next, we investigated the levels of MDA as a lipid peroxidation marker to confirm whether oxidative stress was actually higher in hippocampi subjected to SF, and at 4 days, MDA was 

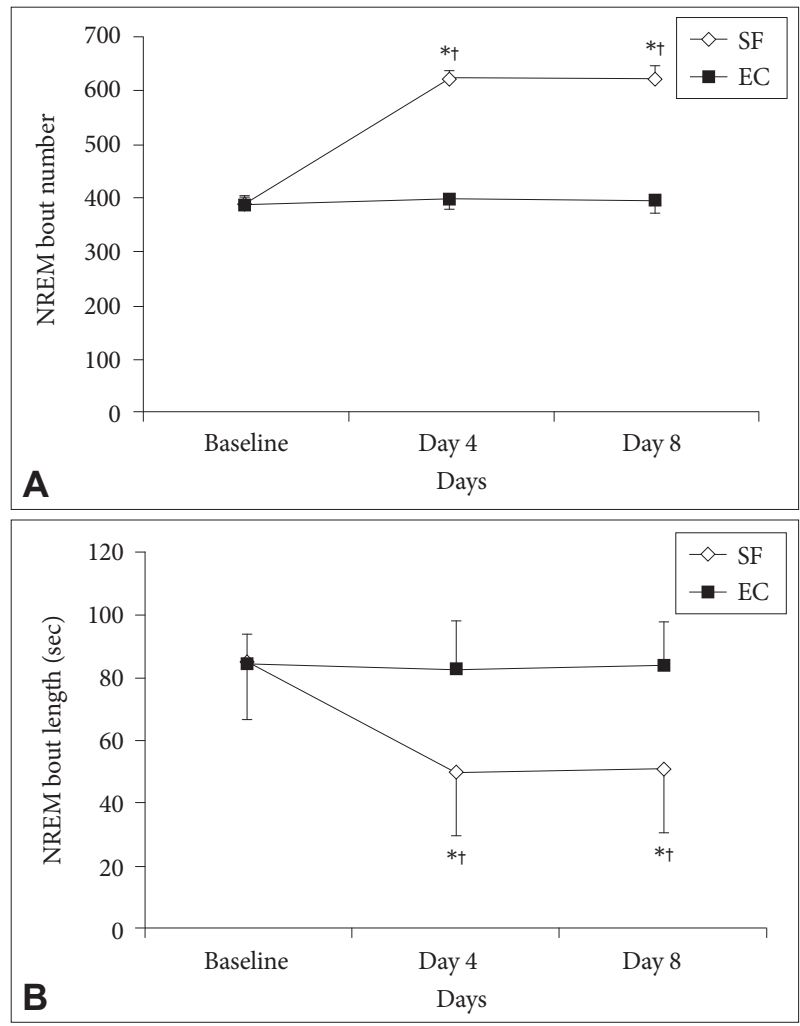

Fig. 2. Mean bout numbers and durations in the sleep-wake stage. A: NREM bout number significantly increased and remained high during the experimental period in the SF group compared with baseline and with the EC group. B: The SF group had shorter NREM bouts at days 4 and 8 than at baseline and shorter bouts than the EC group. The EC group showed no significant changes in NREM bout length or number compared with baseline $\left(\mathrm{n}=5\right.$, each group). Data are expressed as mean \pm SEM. ${ }^{*} \mathrm{p}<$ 0.001 compared with baseline. ${ }^{\dagger} p<0.001$ compared with the EC group. SF: sleep fragmentation, EC: exercise control, NREM: nonrapid eye movement.

higher in the SF group than in the EC group. MDA levels were slightly lower in the 8-day SF group than in the 4-day SF group but still higher than in the 8-day EC group (Fig. 4).

\section{DISCUSSION}

In this study, we investigated the effects of 4 days and 8 days of SF on NLRP-3 activation in rat hippocampi using a forced walking wheel. Four days and 8 days of SF significantly increased NLRP-3 inflammasome expression compared with EC. We also found that higher NLRP-3 inflammasome expression in the SF groups was associated with increased oxidative stress.

Mounting evidence has revealed a significant relationship between sleep disturbance and IL-1 $\beta$. Zielinski et al. ${ }^{18}$ reported that experimentally induced sleep restriction elevated the mRNA expression of IL-1 $\beta$ in several regions of the rat brain. Moreover, according to a recent study that examined the modulating effect

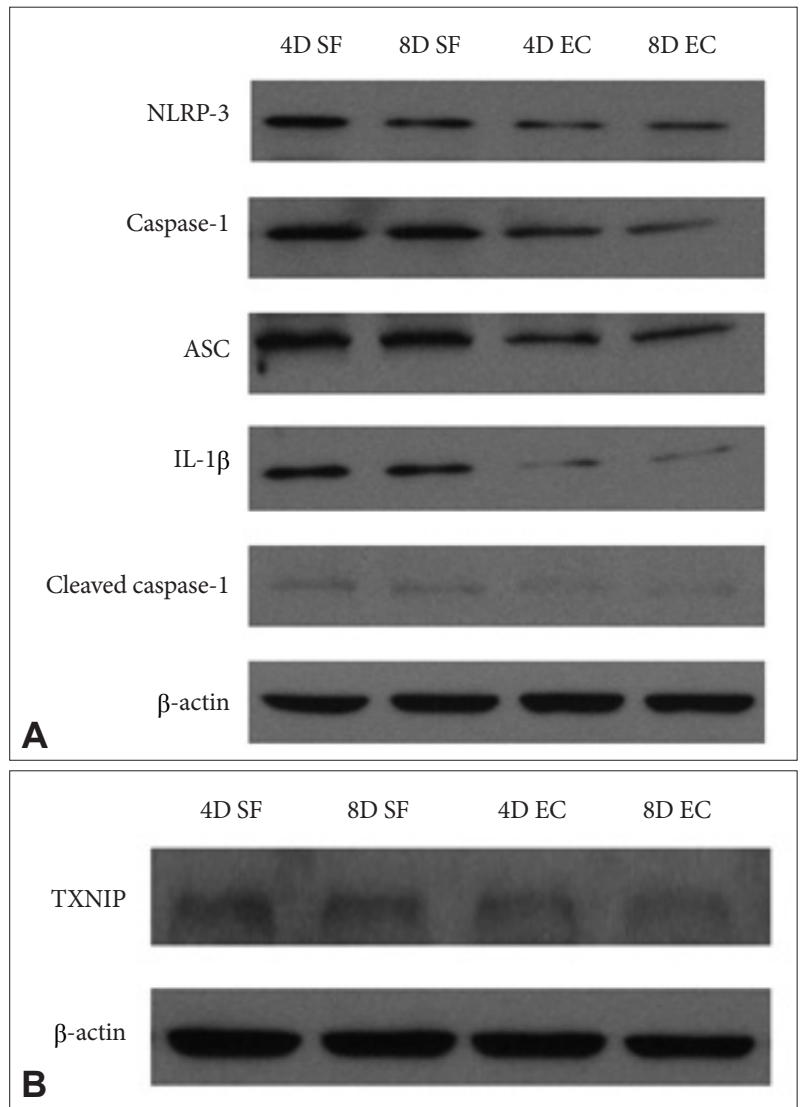

Fig. 3. A: Western blots of the NLRP-3 inflammasome complex (NLRP-3, caspase-1, and ASC), IL-1 $\beta$, and cleaved caspase-1 in the hippocampi of rats exposed to 4 or 8 days of SF or EC $(n=12$, each group). B: Western blots of TXNIP in the hippocampi of rats exposed to 4 or 8 days of SF or EC. SF: sleep fragmentation, EC: exercise control, NLRP-3: NOD-like receptor protein-3, TXNIP: thioredoxin-interacting protein.

of NLRP-3 on spontaneous sleep and homeostatic sleep response following sleep deprivation (SD), NLRP-3 knockout (KO) mice showed no significant differences in IL-1 $\beta$ expression in the somatosensory cortex as well as caspase- 1 activity following 6 $\mathrm{h}$ of SD compared with baseline, whereas wild-type mice showed elevated caspase- 1 activity and NLRP-3, ASC, and IL- $1 \beta$ expression. ${ }^{19}$ In the same study, NLRP-3 KO mice also showed reduced NREM sleep during the light period, and their homeostatic sleep responses following SD were attenuated. These findings suggest NLRP-3 activation as an important molecular mechanism for regulating SD-induced sleep response. Our study is important in that acute SD is known to induce NRLP-3 inflammasome activation in brain regions including the hippocampus, but it is not known in chronic SF conditions, which are more common in real life.

IL-1 $\beta$ has been associated with decreased cognitive function under various conditions; the association between IL-1 $\beta$ levels and hippocampus-dependent memory has a U-shape. Low IL$1 \beta$ promotes long-term potentiation and memory formation, whereas high IL-1 $\beta$ reduces memory consolidation. ${ }^{13,20-22} \mathrm{IL}-1 \beta$ 


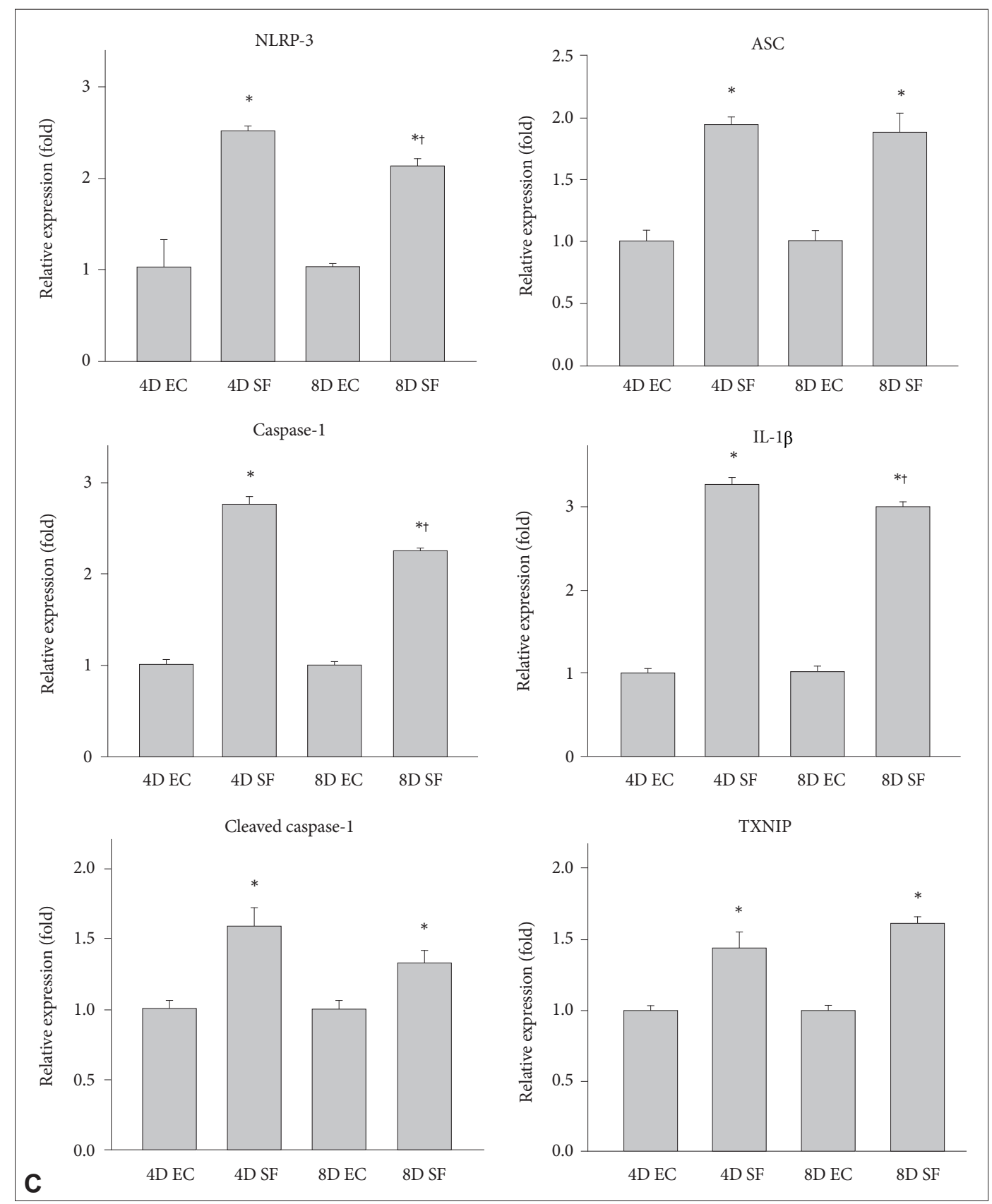

Fig. 3. C: Densitometry results of the Western blot images. Data are expressed as mean \pm SEM. ${ }^{*} p<0.001$ compared with corresponding EC. $t p<0.05$ compared with 4 days of SF. NLRP-3: NOD-like receptor protein-3, SF: sleep fragmentation, EC: exercise control, TXNIP: thioredoxin-interacting protein.

levels have been reported to increase in the hippocampal tissues of obese rats fed high-fat diets, which is associated with memory dysfunction. ${ }^{14}$ However, the same study showed that IL-1 receptor antagonists in the central nervous system restored memory loss, which indicated that IL-1 $\beta$ caused hippocampusdependent memory loss. Several molecular and physiological mechanisms have been suggested to induce hippocampus-dependent memory deficits through IL-1 $\beta$. IL- $1 \beta$ inhibits glutamate release and reduces calcium influx in the hippocampus synaptosome. ${ }^{23}$ It may also affect memory by modulating brain- derived neurotrophic factor (BDNF), which plays an important role in synaptic plasticity and long-term memory. According to a previous study, administering systemic IL-1 $\beta$ or lipopolysaccharide, which can increase IL- $1 \beta$, resulted in down-regulation of BDNF mRNA in rat hippocampal regions subjected to $6 \mathrm{~h}$ of social isolation. ${ }^{24}$ In contrast, an IL-1 receptor antagonist prevented both down-regulation of BDNF expression and memory impairment in rats subjected to social isolation stress. ${ }^{25}$

In addition to a role in memory and cognitive function, NLRP3 inflammasome plays an important role in various metabolic 


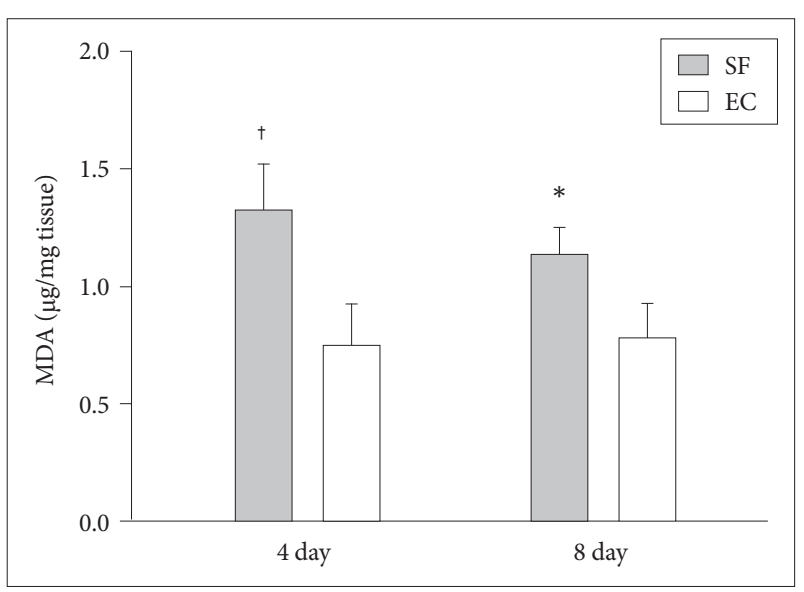

Fig. 4. MDA levels in rat hippocampi exposed to 4 or 8 days of SF or EC ( $n=6$, each group). ${ }^{*} p<0.05$ compared with 8 days of EC. ${ }^{\dagger} p<0.01$ compared with 4 days of EC. SF: sleep fragmentation, EC: exercise control, MDA: malondialdehyde.

diseases. Glucose tolerance and insulin sensitivity have been reported to improve after a high-fat diet was fed to mice that had the NLRP-3 cytoplasmic receptor or ASC and caspase-1 genes knocked out. ${ }^{12}$ NLRP-3 inflammasome may also play an important role in atherosclerosis. Atherosclerosis is a chronic inflammatory disease characterized by the accumulation of lipids and immune cells in blood vessels. ${ }^{26}$ NLRP-3 activation promoted hyperhomocyteinemia-aggravated inflammation in macrophages and concurrent atherosclerosis in apolipoprotein $\mathrm{E}$ (apoE)-deficient mice. ${ }^{27}$ Moreover, inhibiting IL-1 $\beta$ in apoE-deficient mice that had atherosclerosis due to hypercholesterolemia was reported to reduce the incidence of atherosclerotic lesions. $^{28}$

Because previous studies have reported significant associations between sleep disturbance and oxidative stress and because ROS is known to affect NLRP-3 activation, we examined whether NLRP-3 activation in the SF rat groups was associated with oxidative stress. Lipid peroxidation in the hippocampus, as demonstrated by MDA levels, was significantly higher in the SF groups than in the EC groups, indicating increased oxidative stress in the SF groups. In addition, we observed significantly more up-regulation of TXNIP in the SF groups, which binds to NLRP-3 and activates it under oxidative stress. ${ }^{17}$ These findings suggest that SF-induced elevation of oxidative stress activated NLRP-3 inflammasome in rat hippocampi. This possibility could be confirmed pharmacologically by using ROS scavengers.

In this study, we confirmed that SF increased NLRP-3 inflammasome expression and activation in rat hippocampal tissues. Although previous studies have shown that IL-1 $\beta$ plays an important role in hippocampus-dependent memory and cognitive function, this study did not include behavioral tests to assess such cognitive functions. Therefore, future behavioral and mechanistic studies are required to elucidate the role of SFactivated NLRP-3 on hippocampus-dependent cognitive function.

\section{Conflicts of Interest}

The authors have no financial conflicts of interest.

\section{REFERENCES}

1. Kushida CA. Sleep deprivation: basic science, physiology, and behavior. New York: Marcel Dekker 2005.

2. Bonnet MH. Acute sleep deprivation. In: Kryger MH, Roth T, Dement WC. Principles and practice of sleep medicine. 4th ed. Philadelphia, PA: Elsevier-Saunders 2005;59-61.

3. Luyster FS, Teodorescu M, Bleecker E, Busse W, Calhoun W, Castro M, et al. Sleep quality and asthma control and quality of life in non-severe and severe asthma. Sleep Breath 2012;16:1129-37.

4. Berry RB, Gleeson K. Respiratory arousal from sleep: mechanisms and significance. Sleep 1997;20:654-75.

5. Hakim F, Wang Y, Carreras A, Hirotsu C, Zhang J, Peris E, et al. Chronic sleep fragmentation during the sleep period induces hypothalamic endoplasmic reticulum stress and PTP1b-mediated leptin resistance in male mice. Sleep 2015;38:31-40.

6. Zheng J, Almendros I, Wang Y, Zhang SX, Carreras A, Qiao Z, et al. Reduced NADPH oxidase type 2 activity mediates sleep fragmentation-induced effects on TC1 tumors in mice. Oncoimmunology 2015;4: e976057.

7. Choi AJ, Ryter SW. Inflammasomes: molecular regulation and implications for metabolic and cognitive diseases. Mol Cells 2014;37:441-8.

8. Pétrilli V, Papin S, Dostert C, Mayor A, Martinon F, Tschopp J. Activation of the NALP3 inflammasome is triggered by low intracellular potassium concentration. Cell Death Differ 2007;14:1583-9.

9. Martinon F, Pétrilli V, Mayor A, Tardivel A, Tschopp J. Gout-associated uric acid crystals activate the NALP3 inflammasome. Nature 2006; 440:237-41.

10. Halle A, Hornung V, Petzold GC, Stewart CR, Monks BG, Reinheckel T, et al. The NALP3 inflammasome is involved in the innate immune response to amyloid-beta. Nat Immunol 2008;9:857-65.

11. Martinon F. Signaling by ROS drives inflammasome activation. Eur J Immunol 2010;40:616-9.

12. Vandanmagsar B, Youm YH, Ravussin A, Galgani JE, Stadler K, Mynatt $\mathrm{RL}$, et al. The NLRP3 inflammasome instigates obesity-induced inflammation and insulin resistance. Nat Med 2011;17:179-88.

13. Rachal Pugh C, Fleshner M, Watkins LR, Maier SF, Rudy JW. The immune system and memory consolidation: a role for the cytokine IL1beta. Neurosci Biobehav Rev 2001;25:29-41.

14. Sobesky JL, Barrientos RM, De May HS, Thompson BM, Weber MD, Watkins LR, et al. High-fat diet consumption disrupts memory and primes elevations in hippocampal IL-1 $\beta$, an effect that can be prevented with dietary reversal or IL-1 receptor antagonism. Brain Behav Immun 2014;42:22-32.

15. Nair D, Zhang SX, Ramesh V, Hakim F, Kaushal N, Wang Y, et al. Sleep fragmentation induces cognitive deficits via nicotinamide adenine dinucleotide phosphate oxidase-dependent pathways in mouse. Am J Respir Crit Care Med 2011;184:1305-12.

16. Park DS, Yoon DW, Yoo WB, Lee SK, Yun CH, Kim SJ, et al. Sleep fragmentation induces reduction of synapsin II in rat hippocampus. Sleep Biol Rhythms 2014;12:135-44.

17. Zhou R, Tardivel A, Thorens B, Choi I, Tschopp J. Thioredoxin-interacting protein links oxidative stress to inflammasome activation. Nat Immunol 2010;11:136-40.

18. Zielinski MR, Kim Y, Karpova SA, McCarley RW, Strecker RE, Gerashchenko D. Chronic sleep restriction elevates brain interleukin-1 beta and tumor necrosis factor-alpha and attenuates brain-derived neurotrophic factor expression. Neurosci Lett 2014;580:27-31.

19. Zielinski MR, Gerashchenko D, Karpova SA, Konanki V, McCarley RW, Sutterwala FS, et al. The NLRP3 inflammasome modulates sleep and NREM sleep delta power induced by spontaneous wakefulness, sleep deprivation and lipopolysaccharide. Brain Behav Immun 2017;62: 


$$
\text { 137-50. }
$$

20. Avital A, Goshen I, Kamsler A, Segal M, Iverfeldt K, Richter-Levin G, et al. Impaired interleukin-1 signaling is associated with deficits in hippocampal memory processes and neural plasticity. Hippocampus 2003; 13:826-34.

21. Yirmiya R, Goshen I. Immune modulation of learning, memory, neural plasticity and neurogenesis. Brain Behav Immun 2011;25:181-213.

22. Kelly A, Vereker E, Nolan Y, Brady M, Barry C, Loscher CE, et al. Activation of p38 plays a pivotal role in the inhibitory effect of lipopolysaccharide and interleukin-1 beta on long term potentiation in rat dentate gyrus. J Biol Chem 2003;278:19453-62.

23. Plata-Salamán CR, Ffrench-Mullen JM. Interleukin-1 beta depresses calcium currents in CA1 hippocampal neurons at pathophysiological concentrations. Brain Res Bull 1992;29:221-3.

24. Lapchak PA, Araujo DM, Hefti F. Systemic interleukin-1 beta decreases brain-derived neurotrophic factor messenger RNA expression in the rat hippocampal formation. Neuroscience 1993;53:297-301.

25. Barrientos RM, Sprunger DB, Campeau S, Higgins EA, Watkins LR, Rudy JW, et al. Brain-derived neurotrophic factor mRNA downregulation produced by social isolation is blocked by intrahippocampal interleukin-1 receptor antagonist. Neuroscience 2003;121:847-53.

26. Hansson GK. Inflammation, atherosclerosis, and coronary artery disease. N Engl J Med 2005;352:1685-95.

27. Wang R, Wang Y, Mu N, Lou X, Li W, Chen Y, et al. Activation of NLRP3 inflammasomes contributes to hyperhomocysteinemia-aggravated inflammation and atherosclerosis in apoE-deficient mice. Lab Invest 2017 Apr 10 [Epub ahead of print]. https://doi.org/10.1038/labinvest.2017.30.

28. Kirii H, Niwa T, Yamada Y, Wada H, Saito K, Iwakura Y, et al. Lack of interleukin-1beta decreases the severity of atherosclerosis in ApoE-deficient mice. Arterioscler Thromb Vasc Biol 2003;23:656-60. 